\section{Aortic implantation of anomalous left coronary artery}

\section{To the Editor:}

My attention was drawn, rather belatedly, to the excellent article by Laks and associates in the March 1995 issue of the Journal (1995;109:519-23). In this, they described an improved surgical approach to the implantation of the anomalous left coronary artery, which they have used since April 1990. My colleagues and $\mathrm{I}^{1}$ described this technique in a report in The Annals of Thoracic Surgery in 1986, having used it in a baby who had been operated on in 1981. They failed to refer to it.

As I often say to my own junior staff when writing an article, thoroughness of the literature review is one of the basic essentials and would avoid such an omission.

Hugh O'Kane, FRCS

Consultant Cardiac Surgeon

Cardiac Surgical Unit The Royal Hospital

Belfast, Northern Ireland

\section{REFERENCE}

1. Hamilton JRL, Mulholland HC, O'Kane HOJ. Ann Thorac Surg 1986;41:446-8.

[Response declined]

$12 / 8 / 80940$

\section{Use of epsilon-aminocaproic acid to reduce bleeding \\ To the Editor:}

The recent publication by Vander Salm and associates ${ }^{1}$ concerning the use of epsilon-aminocaproic acid (EACA) to reduce bleeding after cardiac operations describes a carefully conducted, randomized, double-blind, placebocontrolled study investigating the efficacy of a high dose of EACA in patients requiring heart surgery. The results show EACA use to be associated with smaller mean volumes of postoperative chest tube drainage than did use of saline solution. This reduction was modest, however, inasmuch as it totaled less than $200 \mathrm{ml}$ per patient over the 24-hour period. The reduction in bleeding did not translate into a clinical benefit, for there was no difference between the EACA group and the placebo group in the transfusion of red blood cells, transfusion of other blood products, or in the mean hematocrit levels of the patients at discharge.

Despite these essentially negative results, the authors have extrapolated from their data the conclusion that "EACA reduces postoperative bleeding after heart operations at far less expense than aprotinin." This is a curious deduction since EACA was not compared with aprotinin in their study; data regarding aprotinin effectiveness are not provided. To conclude that one drug is preferable to another in producing a certain effect, one must compare the two drugs; such a comparison is not provided in the study described by the authors.

In their discussion and conclusions, Vander Salm and associates address only the cost aspects of EACA and aprotinin while ignoring the relative benefits of the two drugs. Cost-benefit comparisons should compare both cost and benefit. EACA certainly costs less than aprotinin, but is it of benefit? The results reported by Vander Salm's group fail to demonstrate any benefit with EACA use, as compared with saline solution, in clinically important parameters such as reduced transfusions of blood products or higher postoperative hematocrit levels. Despite this, without supportive data, the authors state in their discussion that "aprotinin [is] at a considerable disadvantage in the choice between the two drugs on the basis of a cost-benefit analysis." On the basis of their results, however, it appears that EACA is at the disadvantage because no clinically important benefit was associated with its use in this study. No matter how low the cost, without a benefit there is no cost-benefit advantage. To be cost-effective a drug must first be effective.

Several multicenter, randomized, double-blind North American studies involving more than 1375 patients have demonstrated clear-cut aprotinin efficacy in reducing transfusions (not just bleeding) by approximately $50 \%$ as compared with placebo. ${ }^{2}$ Because transfusions are reduced, the cost of aprotinin (about $\$ 450$ for the "half-dose" regimen) is offset, in whole or in part, by the reduction in costs of the blood products themselves, and several studies have demonstrated aprotinin use to be cost-advantageous when the costs of both the blood products and the drug are analyzed..$^{3-5}$ Such considerations were not included in the discussion and conclusions provided by Vander Salm and associates.

I have two further comments:

Vander Salm and associates have referenced the paper by Laub and coworkers ${ }^{6}$ as evidence that aprotinin causes graft closure. They do not point out, however, that the publication by Laub described the single-center results (Deborah Heart and Lung Center) of a small number of patients who participated in a large multicenter trial (University of Iowa, Mayo Clinic, University of Illinois at Chicago, University of Chicago, and Deborah Heart and Lung Center). The results of the multicenter investigation were published earlier ${ }^{7}$ and, with 170 patients and 476 grafts, did not confirm the results of the 32 patients and 81 grafts described by Laub's group. In the multicenter trial, which included the patients reported on later by the Laub's group, an adverse effect on vein graft patency rates was not demonstrated although, as pointed out by the authors, the number of grafts did not provide sufficient statistical power for absolute conclusions in this regard. The reasons for a higher, although not statistically significant, graft closure rate in the aprotinin-treated patients from the single center reported by Laub and associates are not clear.

The "commonly used high-dose aprotinin regimen" described by Vander Salm and colleagues in their discussion is an unusual dose; references to its source are not provided. It does not correspond to the original high-dose (Hammersmith) regimen described by Royston and coworkers ${ }^{8}$ nor to the high-dose, half-dose, or pump-primeonly doses investigated in various published trials. In fact, the dose described by Vander Salm's group is approximately ten times higher than the dose suggested in the 